\title{
Energy Efficient Based Maximal Lifetime Routing in Wireless Sensor Networks
}

\author{
Hua Chen ${ }^{1}$, Min Luo ${ }^{2}$ and Baolin Sun ${ }^{3}$ \\ ${ }^{1}$ College of Mathematics \& Computer Science, Wuhan Textile University, Wuhan \\ 430065, China \\ ${ }^{2}$ School of Information Engineering, Wuhan International Trade University, Wuhan \\ 430205, Hubei Province, P. R. China \\ ${ }^{3}$ School of Information Management, Hubei University of Economics, Wuhan \\ 430205, Hubei Province, P. R. China \\ E-mail: qiuchen_1022@163.com,whicu@189.cn,blsun@163.com
}

\begin{abstract}
Wireless sensor networks (WSNs) are being envisioned for certain applications like habitat monitoring and environmental sensing. Wireless sensors are deployed to achieve network load balancing, prolonging network lifetime, and improving network coverage. To achieve these goals, energy estimation is one of the main constraints and concerns to consider, while deploying wireless sensors in a WSN field. In this paper, we propose an Energy-efficient based Maximal Lifetime Routing Algorithm to prolong lifetime (EMLRA), which is able to dramatically prolong network lifetime while effectively reducing energy consumption. Through an analytical study, we provide guidance on how to choose parameters in our scheme and demonstrate that the scheme is efficient in both network lifetime and energy consumption. Simulation results show that, with the proposed Energy-efficient based Maximal Lifetime Routing Algorithm in WSN energy consumption, network lifetime, energy consumption balance can be improved in most of cases. It is an available approach to routing decision.
\end{abstract}

Keywords: WSN; energy efficient; network lifetime; performance evaluation

\section{Introduction}

A wireless sensor network (WSN) is a deployment of massive numbers of small, inexpensive, self powered devices that can sense, compute and communicate with other devices for the purpose of gathering local information to make global decisions about a physical environment. WSNs are important for a number of pervasive applications such as coordinated target detection, surveillance, and localization. WSNs are a fundamental part of the networking infrastructure for pervasive computing. In such networks, the nodes are often powered by battery, and energy efficient operations are critical to prolong the lifetime of the connections. Designing an energy efficient and Maximal lifetime for such networks is a challenging issue [1-12]. However, for real-time sensing, latency and reliability are of paramount importance, whereas in battery powered sensor networks, energy efficiency is an important metric.

In the WSN, sensor nodes are constrained in energy supply. A sensor node is unable to function while its energy is exhausted. The network being wireless operate on batteries which have limited life. The wireless devices have limited bandwidth and the network provides 
unreliable service resulting in high packet loss and throughput. These networks are not scalable. As a mobile device, the limited battery volume cannot supply the durable power to the sensor node [5], known as power wall. Depending on the applications, the sensors are deployed randomly or using a systematic approach to gather the information from the environment [6]. Wang et al. [7] propose an energy efficient and collision aware (EECA) node-disjoint multipath routing algorithm. The data collection rate in pervasive healthcare systems is high. The development of efficient data and energy processing techniques are of great importance. One of the bottlenecks of sensor devices is the batteries. Considering the likelihood of forgetting to recharge the batteries of several sensors, this is a significant issue to be solved. Although there is much effort on designing low-power sensors to minimize this bottleneck [8], we still need energy scavenging techniques.

To capture the advantages of energy balance, the paper propose a new energy efficient evaluating methodology of packet transmissions in WSNs, which is based on energy efficient, network lifetime and energy consumption balance. The resulting energy efficient and Maximal lifetime routing algorithm is called EMLRA.

The rest of the paper is organized as follows: In Section 2, we briefly review related work. Section 3 presents energy consumption scheme in WSN. Some simulating results are provided in Section 4. Finally, the paper concludes in Section 5.

\section{Related Work}

To save energy, multihop routing is more preferable than sensor-to-sink direct transmission for long-distance transmissions. Research works on energy model of WSN mainly focus on energy model and estimation.

Tariq et al. [9] proposes a comprehensive energy model to estimate the overall energy consumption through power dissipation, for both static and mobile sensors in the potential sensor fields. Wang et al. [10] proposes an energy-efficient transmission scheme for poweradjustable radio to optimize transmit energy efficiency subject to given overflow and delay constraints. An analytical model is developed to estimate the unit energy, data throughput, and delay for a sensor node only in the single-hop case. Gandham et al. [11] proposes to split the lifetime of a sensor network into equal periods of time referred to as rounds and model the energy constrained routing during a round as polynomial time solvable flow problems. In [12] entropy analysis is used for the multi-sensor information fusion process. The proposed entropy method is improves the information transmission by optimizing the fusion process. Rizk et al. [13] proposes a distributed energy balanced algorithm for data routing in WSNs. This method introduces two different cascaded algorithms to prolong the network's lifetime. Liu [14] propose an approach to balance energy consumption and maximize network lifetime for uniformly deployed gradient-sinking sensor networks. Our approach differs from the ones proposed in previous works in that it not only balances the energy consumption among nodes in different gradient, but also balances the energy consumption of nodes in the same gradient. Sinha et al. [15] proposes an energy efficient multi-level aggregation strategy which considers data sensing as continuous stochastic process. This proposed strategy performs filtration of sensed data by removing the redundancy in the sensed data pattern of the sensor node using Brownian motion. Liu et al. [16] proposes an energy efficient routing protocol (BERR) for real-time, reliable communication for wireless sensor networks. It uses the joint performance of real-time, energy efficiency, reliability and the retransmission mechanism with link error rate to choose the next hop node. 
Although network lifetime usually refers to the time interval between the initialization of the network and the exhaustion of the battery of the first sensor node, the practical network lifetime is the time that the network is able to achieve the application requirements.

\section{Energy Consumption Scheme (ECS)}

\subsection{Network Model}

This paper will discard such an unrealistic assumption, and develop a practicable model for WSNs. Given a undirected graph $G(V, E)$ with $n$ nodes and a function state for each node $v \in V$, a source nodes $s \subset V$, and a destination node $d \in V$. Select a set of routing paths for source nodes in order. We will propose the WSN model by using Poisson point process in $\mathbf{R}^{2} \subset G$. We consider a WSN where nodes are randomly distributed within a specified region $B \subset \mathbf{R}^{2}$. Let $\Phi_{0}$ be a Poisson point process in $\mathbf{R}^{2}$ with intensity $\lambda_{0}$ which characterizes the population of the nodes in the region $B$. Hence, there are $\lambda_{0}$ nodes in the WSN in average. Let $\Phi_{1}, \Phi_{2}, \ldots, \Phi_{n-1}$ be $n-12$-dimensional independent Poisson processes with intensities, $\lambda_{1}, \lambda_{2}, \ldots, \lambda_{n-1}$, which are also independent of $\Phi_{0}$. It is assumed that each node is equipped with an omnidirectional antenna and that its transmission power can be adjusted dynamically, to control its transmission range.

\subsection{Energy Consumption Model}

In this section, we derive the energy efficient gains that can be achieved by using EMLRA. We assume that the source node $S$, needs to transmit symbols to the destination node $D$. Without loss of generality, it is assumed that the symbol period for each symbol is normalized to one second.

In a WSN network, each sensor node can operate in a role-dependent (source, destination or router) state that changes temporarily. These states include one of the following:

1. Send packet: node sends data and control packets.

2. Receive packet: node receives data, control and any overheard packets.

3. Forward packets: node receives and sends a particular packet.

4. Idle state: node does not receive or send any packets. This is a rare state in WSNs where routing packets are regularly flooded and overheard by most of the nodes in the network.

If the data rate is $b$ bits/second, to transmit a data packet of $b$ bits over a distance $d$, the corresponding energy consumption for transmission and receiving are as follows;

$$
\begin{gathered}
P_{T x}=\beta_{1} b+\beta_{2} d^{\alpha} b, P_{R x}=\beta_{1} b \\
P=P_{T x}+P_{R x}
\end{gathered}
$$

here, $\beta_{1}=50 \mathrm{~nJ} / \mathrm{bit}$ is the amount of energy dissipated by the radio to run the transmitter or receiver circuitry and $\beta_{2}=100 \mathrm{pJ} / \mathrm{bit} / \mathrm{m}^{2}$ is the transmit amplifier energy. $\alpha$ is the path exponent that indicates the rate at which the path loss increases with distance. 


\subsection{Energy Consumption Analysis}

In this section, we have performed the analysis of energy consumed by the sensors in the network. In the analysis, we have considered the energy consumed in packets communication as well as computations required for data aggregation. $N$ sensor nodes are distributed according to two dimensional Poisson process with intensity $\lambda$ in squared sensing region with each side $2 a$ units. Hence the area $\left(\mathbf{R}^{2}\right)$ of the sensing region becomes $4 a^{2}$ square units.

Now, we calculate the energy consumption of a wireless sensor node $x$ (i.e. amount of energy used in one second). Every sensor node is supposed to consist of a set of member nodes that only involves in sampling and transmitting the aggregate of sampled data. The radio propagation within a sensor node is assumed to free space, i.e. the transmission signal attenuates over the square of distance towards the sensor node. The energy used in transmission for sending $b$ bits of packet to the node $x$ is:

$$
P_{T x}(b, d)=b\left(P_{R x}+\varepsilon_{f x} d^{2}\right)
$$

where, $\varepsilon_{f x}$ signifies the energy used by the amplifier module.

The expected number of hops from two sensors node located at $\left(x_{1}, y_{1}\right)$ and $\left(x_{2}, y_{2}\right)$, having distance $d$, and communication range $r$, is $[d / r]$. The boundary is given by:

$$
\left(\frac{d}{r}\right) \leq\left(\left\lceil\frac{d}{r}\right\rceil\right)<\left(\frac{d}{r}+1\right)
$$

The energy consumed through multi-hop communication between a source node $S$ and a destination node $D$ with distance $d$ apart, and has communication range $r$, is given by:

$$
\left(P_{T x}+P_{R x}+\varepsilon_{f x} \square r^{\beta}\right)\left(\frac{d}{r}\right)
$$

Now, we estimated distance $E\left[d^{2}\right]$ between the wireless sensor node $x$ and the other nodes $y$ for Poisson Distributed nodes.

$$
E\left[d^{2}\right]=\int_{R^{2}}\left[\left(x-c_{x}\right)^{2}+\left(y-c_{y}\right)^{2}\right] \cdot \phi_{p o s n} \cdot d R^{2}
$$

We can determine the total power dissipation of the WSNs at time $t$, as:

$$
P_{\text {total }}(t)=\sum_{i=1}^{n}\left(P_{T x}+P_{R x}+\varepsilon_{f x} \square r^{\beta}\right)
$$

Energy consumed through data communication is given by:

$$
E_{\text {cons }}(t)=\left(P_{T x}+P_{R x}+\varepsilon_{f x} \square r^{\beta}\right)\left(\frac{0.521 \square R}{r}+0.5\right) \square \lambda T_{\text {data }}(t)
$$

where, $T_{\text {data }}(t)$ is the total time $t$ a sensor node $x$ is involved in data communication. Therefore, total energy consumption $E_{\text {total }}$ can be calculated from (8) as:

$$
E_{\text {total }}=\sum_{i=1}^{n}\left(\left(P_{T x}+P_{R x}+\varepsilon_{f x} \square r^{\beta}\right)\left(\frac{0.521 \square^{R}}{r}+0.5\right) \square \lambda \square T_{\text {data }}(i)\right)
$$




\section{Simulation Experiments}

In this section, we evaluate the performance of the proposed algorithms against the existing algorithms through the simulations. We consider a network of randomly distributed nodes within a specified square area. The source node and the destination nodes are located in the diagonal corners of the network.

\subsection{Simulation Model and Performance Metrics}

To conduct the simulation studies, we have used randomly generated networks on which the algorithms were executed [17]. This ensures that the simulation results are independent of the characteristics of any particular network topology.

The sensing area is assumed to be a square region with dimension $1000 \times 1000 \mathrm{~m}^{2}$. The sensors are Poisson distributed and initially have identical communication radius of $250 \mathrm{~m}$. Its initial value corresponds to the node energy level at the beginning of the simulation. We also assumed that the energy required to receive a message is the sum of the fixed cost of powering the antenna plus the fixed processing cost. When the node energy level goes down to zero, the node dies out, that is no more packets can be received or transmitted by the node. The initial energy of each node battery is $30 \mathrm{~J}$ in the reference scenario.

To effectively evaluate EMLRA's performance, we compare it with other famous multipath routing protocols, EECA [7], and BERR [16] for cost to control information, average link-connect time, the success rate to find the path and the feature of data transmission. Table 1 lists the simulation parameters which are used as default values unless otherwise specified.

Table 1. Simulation Parameters

\begin{tabular}{|c|c||c|c|}
\hline Number of nodes & 100 & Correct receive threshold & $3.7 \times 10^{-10} \mathrm{~W}$ \\
\hline Terrain range & $\begin{array}{c}1000 \mathrm{~m} \\
\times 1000 \mathrm{~m}\end{array}$ & $\begin{array}{c}\text { Threshold to avoid } \\
\text { collisions }\end{array}$ & $1.6 \times 10^{-11} \mathrm{~W}$ \\
\hline Transmission range & $250 \mathrm{~m}$ & Channel bandwidth & $1-3 \mathrm{Mbps}$ \\
\hline Average node degree & $3-5$ & Links delay & $20-200 \mathrm{~ms}$ \\
\hline Simulation time & $600 \mathrm{~S}$ & Traffic type & $\mathrm{CBR}$ \\
\hline $\begin{array}{c}\text { Maximum transmit power } \\
T_{t}^{\text {max }}\end{array}$ & $0.25 \mathrm{~W}$ & Node pause time & $10 \mathrm{~S}$ \\
\hline Initial node energy & $30 \mathrm{~J}$ & $\begin{array}{c}\text { Examined routing } \\
\text { protocol }\end{array}$ & $\begin{array}{c}\text { EECA and } \\
\text { BERR }\end{array}$ \\
\hline
\end{tabular}

We will compare the performance of three multipath routing methods under the same energy model and communication models. Performance metrics we have used in our experiments are energy consumption, packet delivery ratio (PDR), and network lifetime.

1. Energy consumption: Energy consumption is measured at the radio layer during the simulation based on the specification of IEEE 802.11- WLAN. The energy consumption varies from 0.013 Watt in a low-power sleep state to $0.83,1.0$, and 1.4 Watt in idle listening, receiving, and transmitting states, respectively.

2. Network lifetime: The network lifetime is defined as the smallest time that it takes for at least one node in the network to drain its energy.

3. Energy Consumption Balance (ECB): Defined as the ECB metric to determine the effectiveness of the energy balancing algorithm used by wireless sensor node 
mechanism. If the ECB for each node $n$ is $E_{n}$, the relationship between the ECB of node $n$ and the total consumed energy in all the network's nodes is:

$$
E_{n}(\mathrm{ECB})=E_{\text {n-consumed }} / E_{\text {total-consumed }}
$$

\subsection{Simulation Results}

The results of the simulation are positive with respect to performance. We use the Network Simulator version 2 (NS-2) [18] to evaluate the EMLRA protocols. NS-2 is a discrete event simulator targeted at networking research. NS-2 provides substantial support for simulation of TCP, routing, and multipath protocols over wired and wireless networks.

In the sequel, we consider the impact of the number of nodes on the energy saving gain expression in (8), (9). The number of node is in the range of 10 to 100 in WSNs. Figure 1 compares the energy consumption of the EMLRA algorithm with EECA and BERR. We observe that energy efficient outperforms the other schemes: it achieves the lowest energy consumption for most of the number of nodes. Multi-path forwarding achieves a similar energy consumption and a lower successful delivery ratio than energy efficient.

The most significant improvement of protocol EMLRA is to prolong the network lifetime with balancing the energy consumption and enhancing energy available. Figure 2 show the network lifetime of EMLRA and other comparative protocols under different node numbers and packet numbers respectively. It clearly shows the increase in network lifetime with the proposed algorithm EMLRA with varying threshold probability as compared to existing EECA, and BERR routing protocol. While, to enhance the network lifetime, EMLRA considers the sender's energy efficiency and neighbors' energy efficiency, when transmit a data packet from the current node to sink node.

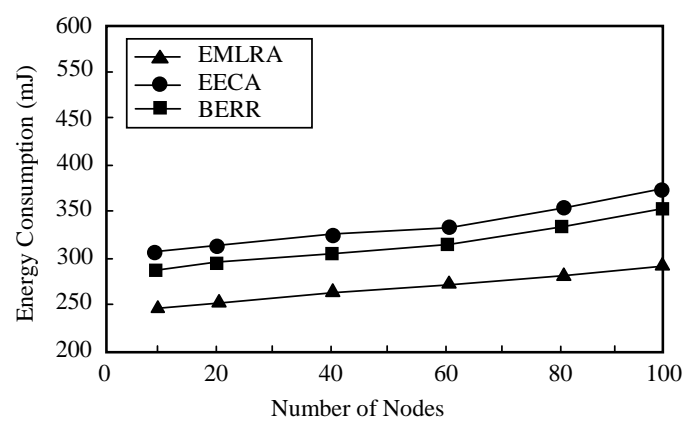

Figure 1. Comparison of Energy Consumption

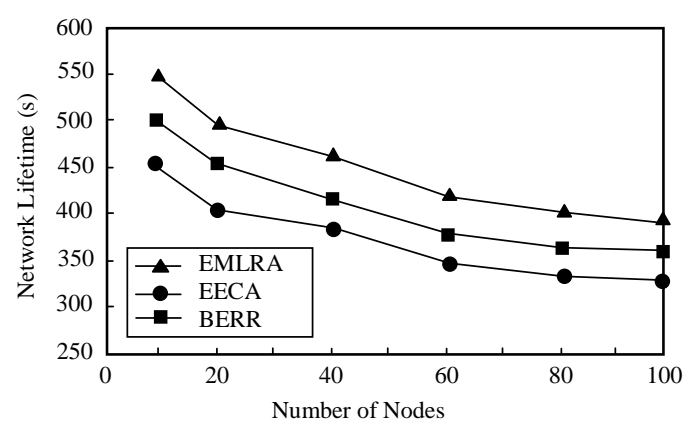

Figure 2. Comparison of Network Lifetime

The most important metric of our performance evaluation is energy consumption. For each approach, we measure the total energy consumption that all source nodes successfully send data packets to sink. Fig.3 shows EMLRA is the most energyefficient solution.

The main characteristic of our protocol EMLRA is to enhance energy efficiency by balancing energy consumption among the nodes. Figure 4 above shows the average value for the EMLRA of each node over the duration of the simulation. The EMLRA indicates the variation in Energy consumption of nodes as a fraction of total energy consumed in the network. Therefore, the fraction denotes the energy consumption load 
per node compared to total energy load and demonstrates the ratio of energy that each node has consumed during its operation. It can be seen from this Figure 4 that in the case of EMLRA, the energy consumption is spread more evenly across the nodes of the network as compared to both EECA and BERR.

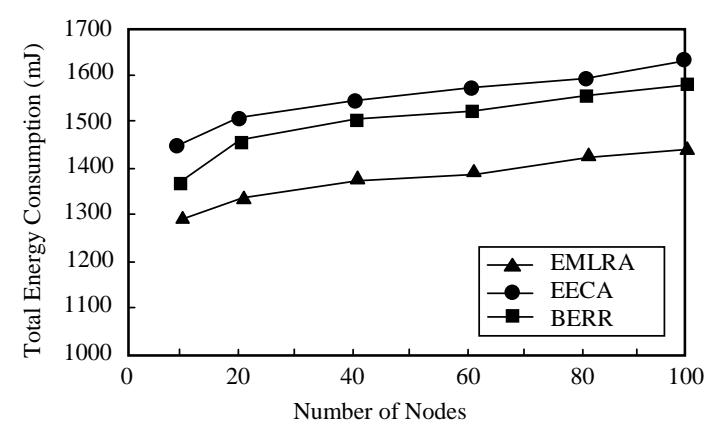

Figure 3. Total Energy Consumption According to the Number of Nodes

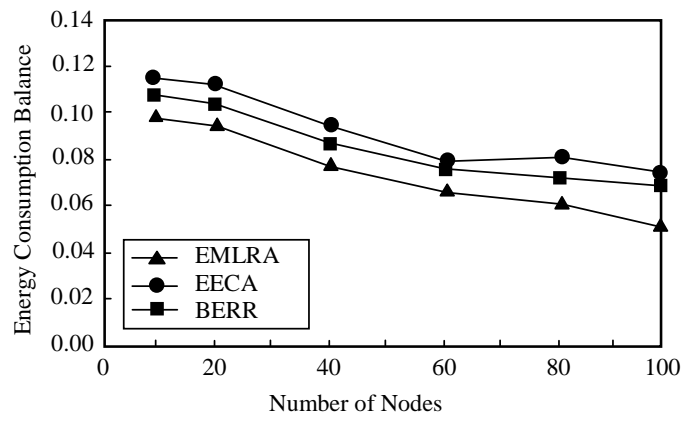

Figure 4. The Average Value of Energy Consumption Balancing

\section{Conclusion}

In this paper, we introduced an energy model for estimating energy consumption through power dissipation for both static as well as sensors node in WSNs.

This model considers all aspect of energy consumption through power dissipation, by deploying the sensors. In this paper, we propose an Energy-efficient based Maximal Lifetime Routing Algorithm to prolong lifetime (EMLRA), which is able to dramatically prolong network lifetime while effectively reducing energy consumption. Last, we evaluate the performance of various schemes through simulation. The simulation results confirm the analytical study that our scheme is efficient in energy consumption, network lifetime, energy consumption balance in WSNs.

\section{Acknowledgements}

This work is supported by The Young and Middle-aged Elitists' Scientific and Technological Innovation Team Project of the Institutions of Higher Education in Hubei Province under Grant No. T200902. Natural Science Foundation of Hubei Province of China under Grant No. 2013CFB035, 2013CFB309. Key Project of Hubei Province Education Science During the 12th Five-Year under Grant No. 2012A042.

\section{References}

[1] B. L. Sun, C. Gui and Y. Song, "Stable Clusterhead Selection Algorithm for Ad Hoc Networks", International Journal of Future Generation Communication and Networking, vol. 6, no. 3, (2013), pp. 95-106.

[2] B. L. Sun, S. C. Pi and C. Gui, "Multiple Constraints QoS Multicast Routing Optimization Algorithm in MANET based on GA", Progress in Natural Science, vol. 18, no. 3, (2008), pp. 331-336.

[3] B. L. Sun, C. Gui, and Y. Song, "Energy Entropy On-Demand Multipath Routing Protocol for Mobile Ad Hoc Networks", China Communications, vol. 8, no. 7, (2011), pp. 75-83.

[4] B. L. Sun, C. Gui, Y. Song, and H. Chen, , "A Novel Network Coding and Multi-path Routing Approach for Wireless Sensor Network", Wireless Personal Communications, online: DOI 10.1007/s11277-013-1496-y

[5] M. Qiu, C. Xue, Z. Shao, M. Liu, and E. H.-M. Sha, "Energy minimization for heterogeneous wireless sensor networks", Journal of Embedded Computing, vol. 3, no. 2, (2009), pp. 109-117.

[6] S. Choudhury, S. Akl and K. Salomaa, "Energy efficient cellular automaton based algorithms for mobile wireless sensor networks", in Proceedings of IEEE Wireless Communications and Networking Conference (WCNC '12), (2012), April, pp. 2341-2346. 
[7] Z. J. Wang, E. Bulut and B. K. Szymanski, "Energy Efficient Collision Aware Multipath Routing for Wireless Sensor Networks", In Proceedings of IEEE ICC, (2009), pp. 1-5.

[8] J. Yoo, L. Yan, S. Lee, Y. Kim, and H.-J. Yoo, "A $5.2 \mathrm{~mW}$ self-configured wearable body sensor network controller and a $12 \mu \mathrm{W}$ wirelessly powered sensor for a continuous health monitoring system", IEEE Journal of Solid-State Circuits, vol. 45, no. 1, (2010), pp. 178-188.

[9] M. Tariq, M. Macuha, Y. J. Park and T. Sato, "An energy estimationmodel formobile sensor networks," in Proceedings of the 4th International Conference on Sensor Technologies and Applications (SENSORCOMM'10), (2010), July, pp. 507-512.

[10] C. Wang and P. Ramanathan, "Energy efficient transmission scheme for data-gathering in mobile sensor networks," in Proceedings of the 3rd Annual IEEE Communications Society on Sensor and Ad hoc Communications and Networks (Secon'06), vol. 2, (2006) September, pp. 498-507.

[11] S. Gandham, M. Dawande and R. Prakash, "An integral flowbased energy-efficient routing algorithm for wireless sensor networks", Proceedings of IEEE Wireless Communications and Networking Conference (WCNC'04), vol. 4, (2004), pp. 2341-2346.

[12] P. Xie and Y. H. Du, "Study on an information fusion method based on information entropy and wavelet neural network', In it IEEE fourth international conference on natural computation, (2008), pp. 354-357.

[13] M. R. M. Rizk, M. A. Abdou and S. S. A. El-Dayem, "An optimum distributed energy balanced algorithm for wireless sensor networks", Alexandria Engineering Journal, vol. 52, no. 1, (2013), pp. 13-18.

[14] T. Liu, "Avoiding Energy Holes to Maximize Network Lifetime in Gradient Sinking Sensor Networks", Wireless Personal Communications, vol. 70, no. 2, (2013), pp. 581-600.

[15] A. Sinha and D. K. Lobiyal, "A Multi-Level Strategy for Energy Efficient Data Aggregation in Wireless Sensor Networks", Wireless Personal Communications, vol. 72, no. 2, (2013), pp. 1513-1531.

[16] Z. X. Liu, L. L. Dai, K. Ma and X. P. Guan, "Balance energy-efficient and real-time with reliable communication protocol for wireless sensor network", The Journal of China Universities of Posts and Telecommunications, vol. 20, no. 1, (2013), pp. 37-46.

[17] B. Waxman, "Routing of Multipoint Connections", IEEE Journal on Selected Areas in Communications, vol. 6, no. 9, (1988), pp. 1617-1622.

[18] The Network Simulator - NS-2, http://www.isi.edu/nsnam/ns/.

\section{Authors}

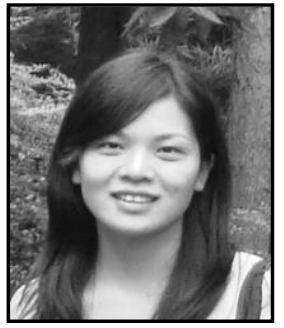

Hua Chen received his M.S. degree from Wuhan University, China in 2005. She is currently an associate professor of mathematics and computer science of Wuhan Textile University, China. Her research interests include algorithm, wireless communication, performance analysis, and network model. She has published over 20 research papers. E-mail: qiuchen_1022@163.com.

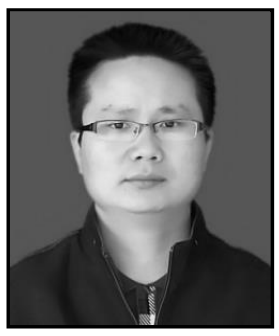

Min Luo received his M.S. degree from Wuhan University, China in 2003. He is currently a lecturer in computer science and technology of Wuhan International Trade University, China. His research interests include wireless communication and network optimization. E-mail: whicu@189.cn.

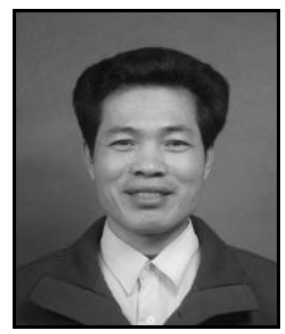

Baolin Sun received his Ph.D. degree from Wuhan University of Technology, China in 2006. He is currently a professor in computer science and technology of Hubei University of Economics, China. His research interests include multipath routing, parallel and distributed computing, network optimization and ad hoc networks. He has published over 120 journal and conference papers and has author of four books in the above areas. E-mail: blsun@163.com. 